\title{
Semaphorin signaling restricts neuronal regeneration in C. elegans
}

Harreguy $\mathrm{MB}^{1,2}$, Shah $\mathrm{E}^{1}$, Tanvir $\mathrm{Z}^{1,2}$, Simprevil $\mathrm{B}^{1}$, Tran $\mathrm{TS}^{2}$, Haspel $\mathrm{G}^{1 *}$

${ }^{1}$ New Jersey Institute of Technology, Department of Biological Sciences, Newark, New Jersey, USA

${ }^{2}$ Rutgers University, Department of Biological Sciences, Newark, New Jersey, USA

\section{* Correspondence:}

haspel@njit.edu

Key Words: neuroregeneration, semaphorins, plexins, laser microsurgery, C. elegans, locomotion

\begin{abstract}
Extracellular signaling proteins serve as neuronal growth cone guidance molecules during development and are well positioned to be involved in neuronal regeneration and recovery from injury. Semaphorins and their receptors, the plexins, are a family of conserved proteins involved in development that, in the nervous system, are axonal guidance cues mediating axon pathfinding and synapse formation. The Caenorhabditis elegans genome encodes for three semaphorins and two plexin receptors: the transmembrane semaphorins, SMP-1 and SMP-2, signal through their receptor, PLX-1, while the secreted semaphorin, MAB-20, signals through PLX-2. Here, we determined the neuronal morphology and locomotion behavior of knockout animals missing each of the semaphorins and plexins; we described the expression pattern of all plexins in the nervous system of $C$. elegans; and we evaluated their effect on the regeneration of motoneuron neurites and the recovery of locomotion behavior following precise laser microsurgery.
\end{abstract}

\section{Introduction}

During neurodevelopment, growth factors and guidance cues regulate dendrite morphogenesis, axon growth cone initiation and navigation, axon elongation and target recognition, but their effects are less pronounced in the adult nervous system. Studying their role in the context of adult regeneration and recovery could provide insight into the molecular and cellular response to injury (Chen et al., 2011; Chisholm et al., 2016).

The semaphorins are a family of glycosylated proteins that were first characterized for their role in the development of the insect and avian nervous systems as axonal guidance cues but were later found in a variety of other tissues and organisms (Alto and Terman, 2017; Junqueira Alves et al., 2019). All semaphorins have a distinctive 500 residue long $\mathrm{N}$-terminal domain, known as the Sema domain. This domain, which is a seven-blade beta-propeller, with each blade formed by four anti-parallel beta-strands (Gherardi et al., 2004), is exclusive to semaphorins and their receptors, the plexins, 
where it mediates semaphorin dimerization and receptor binding. Eight classes of semaphorins are phylogenetically conserved in nematodes, flies, chick, mammals, and viruses, with three classes of smaller proteins that are secreted and five classes that are membrane-bound by a transmembrane domain or a glycosylphosphatidylinositol (GPI) link (Alto and Terman, 2017; Junqueira Alves et al., 2019). Correspondingly, four classes of plexins are conserved in invertebrates and vertebrates (Tamagnone et al., 1999; Negishi et al., 2005). All plexins are transmembrane proteins with an extracellular Sema domain that mediates semaphorin binding and signaling, either by themselves or with a neuropilin co-receptor, in the case of the secreted class 3 semaphorins in vertebrate (Negishi et al., 2005; Pascoe et al., 2015).

In mammals, semaphorins and their receptors, neuropilins and plexins, were originally described as guidance cues for neuronal growth cones aiding axons to their targets by acting as chemorepellents (Kolodkin and Tessier-Lavigne, 2011). More recently, semaphorins have been implicated in multiple key roles of neural circuit assembly during neurodevelopment (Yoshida, 2012; Koropouli and Kolodkin, 2014). For example, the mammalian secreted semaphorin, SEMA3A, is involved in various

neurodevelopmental processes in the mouse, including repelling dorsal root ganglion sensory axons, promoting basal dendrite elaboration in cortical pyramidal neurons, and pruning of hippocampal axons (Bagri et al., 2003; Yaron et al., 2005; Mlechkovich et al., 2014; Danelon et al., 2020). Another well studied secreted semaphorin, SEMA3F, and its receptor Neuropilin-2, are also involved in axon guidance, synaptic plasticity, and refinement, as well as in restraining the excess of dendritic spines on apical dendrites of cortical neurons and regulating inhibitory interneuron numbers in the hippocampus (Tran et al., 2009; Riccomagno et al., 2012; Riccomagno and Kolodkin, 2015; Assous et al., 2019; Eisenberg et al., 2021). As the mediators of semaphorin signaling, the plexins are involved in axon guidance, synapse and dendrite formation, axonal pruning and synaptic stability (Shen and Cowan, 2010; Limoni, 2021).

In accordance with their role in neurodevelopment, semaphorins could be involved in axonal regeneration after injury (Fard and Tamagnone, 2021). For example, SEMA3A expression levels increase after injury in the spinal cord and cerebral cortex (de Winter et al., 2002; Hashimoto et al., 2004) and regenerating axons avoid areas with high SEMA3A expression (Pasterkamp and Verhaagen, 2001). Accordingly, a SEMA3Aspecific inhibitor improved axon regeneration and spontaneous hind leg movement after spinal cord transection (Kaneko et al., 2006). Plexin expression and function in response to injury varies depending on the type. Plexin A family members increase their expression after axonal injury in facial motoneurons and rubrospinal neurons contributing to the role of semaphorins in restricting regeneration (Spinelli et al., 2007). On the other hand, PlexinB2 is upregulated after spinal cord injury in glial cells proximal to the injury site and is required for wound healing and recovery (Zhou et al., 2020).

The Caenorhabditis elegans genome encodes for only three semaphorin and two plexin homologues. Of those, PLX-1 binds the two membrane-bound semaphorins (SMP-1 and SMP-2), while PLX-2 binds the only secreted semaphorin (MAB-20; Figure 1A; (Ginzburg et al., 2002; Nakao et al., 2007). Both membrane-bound and secreted semaphorin-plexin systems are involved in development; semaphorins guide ventral enclosure (Ikegami et al., 2012), and regulate epidermal morphogenesis (Ginzburg et 
al., 2002; Ikegami et al., 2012) as well as vulva and tail-rays morphogenesis in the hermaphrodite and males, respectively (Dalpe et al., 2012). In the nervous system, membrane-bound semaphorin signaling (the $p / x-1 / s m p-1 / s m p-2$ pathway) is necessary for synaptic tiling in two DA motoneurons in the tail (Mizumoto and Shen, 2013) and for guidance of the long axons of mechanosensory neurons (Ginzburg et al., 2002). Secreted semaphorin signaling (via the plx-2/mab-20 pathway) contributes to motoneuronal axon guidance; eliminating this pathway, when not embryonic lethal, causes defasciculation of the ventral nerve cord (VNC; $17 \%$ of surviving mab-20 knockout animals) and axon misguidance in DA and DB motoneuron classes (4\% of surviving mab-20 knockout animals;(Roy et al., 2000).

C. elegans is a well-established model for neuronal regeneration and most of its neurons are able to regenerate after precise laser microsurgery (60\%) and in some cases reestablish functional connections (Yanik et al., 2004; Ghosh-Roy and Chisholm, 2010; Neumann et al., 2011; Harreguy et al., 2021). Here we take advantage of the small number of plexins in $C$. elegans and the capability to precisely disconnect single neurites in intact animals, to investigate the role of semaphorin signaling in neuroregeneration in vivo. We describe the neuronal expression of the plexin receptors and the effect of their absence on neuronal regeneration and recovery of locomotion behavior.

\section{Methods}

\section{Strains and transgenics}

We maintained $C$. elegans strains under standard laboratory conditions on nematode growth medium (NGM) agar plates with OP-50-1 Escherichia coli bacterial lawn at $15^{\circ} \mathrm{C}$ (Stiernagle, 2006). All animals used in the experiments were hermaphrodites.

We acquired semaphorin and plexin mutants from Caenorhabditis Genetics Center (CGC) or the C. elegans National Bioresource Project of Japan (NBRP): ev778 (mab20, null), tm729 (p/x-2, null), ev715 (smp-1, null), ev709 (smp-2, null), tm10697 (plx-1, null), and evls111 ([F25B3.3::GFP + dpy-20(+)], pan-neural GFP expression). To allow imaging and microsurgery, we crossed males of NW1229 (evls111), induced by 10 minute exposure of L4 larvae to $10 \%$ ethanol (Lyons and Hecht, 1997), with null-mutant hermaphrodites to obtain knockout animals expressing GFP in the entire nervous system: TOL55 (ev715, evls111), TOL57 (ev709, evls111), TOL59 (tm10697, evls111), and TOL62 (tm729, evls111).

We generated transgenic strains that express EGFP under the $p / x-1 p$ promoter extrachromosomal array. We injected the gonads of adult wild type animals (N2) with a mixture consisting of $2.5 \mathrm{ng} / \mu \mathrm{l}$ pCFJ90 [myo-2p::mCherry], $10 \mathrm{ng} / \mu \mathrm{l}$ of pMS80 [p/x$1 p:: G F P(65 C)$ ] and $87.5 \mathrm{ng} / \mu \mathrm{l}$ of $1 \mathrm{~Kb}$ DNA Ladder (New England Biolabs). We identified insertion by selecting animals expressing mCherry and EGFP. The reporter construct was a generous gift from Dr Kota Mizumoto (University of British Columbia). The reporter strain for $p / x-1 p:: E G F P$ (NW2339) and $p / x-2 p:: G F P$ (NW1693) were shared by Dr Dr. Joseph Culotti (University of Toronto, Mt Sinai Hospital) and Dr Richard Ikegami (UC Berkeley), respectively. 


\section{Locomotion analysis}

We tracked locomotion behavior of multiple animals over an agar surface $(1.7 \%$ in NGM buffer), without food, as well as in liquid (NGM buffer). We recorded videos with a static multi-worm tracker, composed of three major parts, from top to bottom: (1) a CMOS camera (acA4024-29um, Basler) mounted with a fixed focal length lens (C Series 5MP $35 \mathrm{~mm} \mathrm{2/3",} \mathrm{Edmund} \mathrm{Optics),} \mathrm{and} \mathrm{an} \mathrm{infrared} \mathrm{cut-off} \mathrm{filter} \mathrm{(SCOTT-KG3} \mathrm{M25.5 \times 0.5,}$ Edmund Optics); (2) a specimen stage for plates or slides; (3) a collimated Infrared LED light source (M850L3 and COP1-B, Thorlabs).

One day before the experiment, we transferred animals of the fourth larval stage (L4) onto a new plate with healthy OP-50-1 bacterial lawn. Immediately before tracking, animals were transferred onto a $30 \mathrm{~mm}$ agar plate with no food or a 150 ul drop of NGM buffer, placed on a microscope slide. During tracking, animals moved freely, and we recorded multiple $25 \mathrm{~Hz}$ 15-second videos using Pylon Viewer (Pylon Camera Software Suite, Basler). We analyzed the videos with Tierpsy worm-tracker (Javer et al., 2018) that can track multiple animals and extract up to 726 features for each tracked trajectory. We analyzed the HDF5 output file produced by the Tierpsy in Matlab (script code available upon request) to collect the mean speed and frequency values for each trajectory and then plotted the data and estimated confidence intervals among groups with a freely available software for Estimation Statistics (https://www.estimationstats.com; (Ho et al., 2019). We considered neurites regenerated when a new branch was observed extending from the proximal segment of the injury site (Harreguy et al., 2020). When the regenerating branch extended to the distal segment or the target of the pre-injury neurite we considered it reconnected. We tracked all the knockdown, transgenic, and wild type strains without injury to assess their baseline locomotion parameters. Further, 6 and 24 hours after microsurgery, we tracked locomotion to assess recovery. For comparison, we also quantified locomotion parameters of sham-surgery groups for each genotype and time point. We treated the sham-surgery groups through the same protocol (including cooling and immobilization, see below), except for the exposure to the laser beam.

\section{Expression and neuronal morphology analysis.}

We immobilized animals by placing them in a drop of cold, liquid $36 \%$ Pluronic F-127 (thermo-responsive material that gels at room temperature, Sigma-Aldrich) with $1 \mathrm{mM}$ levamisole (a paralytic nicotinic agonist, Sigma-Aldrich), mounted on a microscope slide with a \#1.5 coverslip and sealed with a room-temperature-hardening mixture (VALAP, equal weights of petroleum-jelly, lanolin, and paraffin). We used a laser scanning confocal microscope (Leica SP8; microscope: DM6000CS; objectives: Leica 40x/NA1.30 HC PL APO oil or Leica 63x/NA1.40 HC PL APO oil, with lateral resolutions of $223 \mathrm{~nm}$ and $207 \mathrm{~nm}$ respectively; laser lines: $405 \mathrm{~nm}, 561 \mathrm{~nm}$, and $488 \mathrm{~nm}$ ). We collected multiple optical slices (thickness optimized by the confocal software, ranging $0.343-0.345 \mu \mathrm{m}$ for the $63 \mathrm{X}$ objective, and $0.410-0.422 \mu \mathrm{m}$ for the $40 \mathrm{X}$ objective). To analyze morphology and expression we constructed the maximum intensity projections for at least 10 animals of each strain and, in some cases, processed images to reduce background noise via the Leica Application Suite (LASX) software. 


\section{Laser Microsurgery}

For laser microsurgery and associated microscopy, we mounted C. elegans hermaphrodites at L4 stage by placing them in a drop of ice cold, liquid 36\% Pluronic F127 with $1 \mathrm{mM}$ levamisole solution and pressed them between two \#1 coverslips (Melentijevic et al., 2017). We brought the slides to room temperature, to solidify the Pluronic F-127 gel and immobilize the animals. We used a Yb-fiber laser (100 pulses at $10 \mathrm{kHz}$ repetition rate) to cut a single neurite with submicron precision and no discernable collateral damage (Harreguy et al., 2020). We took images immediately before and after the lesion to visually verify the microsurgery. In some cases, multiple laser exposures were necessary to disconnect a neurite. We disconnected the ventraldorsal commissures of all motoneurons that we were able to identify by their relative position, at about $45 \mu \mathrm{m}$ away from the VNC. We assessed neuronal regeneration 24 hours after microsurgery on the same microscope and imaging system in at least 6 neurons per animal in at least 15 animals for each condition. We classified neurite regeneration in three categories: no regeneration (gap at the injury site, no new branches), regeneration (a new branch extended from the proximal site of lesion), and reconnection (a new branch fused with the distal side of the lesion or with the dorsal nerve cord). We used Image J (FIJI v.1.52) and LASX (Leica) for image processing and visualization, and Prism (GraphPad v.9.2.0) for statistical analysis and plotting.

\section{Results}

\section{C. elegans animals that do not express semaphorins or plexins exhibited altered locomotion patterns.}

We analyzed the contribution to locomotor behavior of each of $C$. elegans three semaphorins and two plexins (Fig. 1A) by observing knockout (ko) mutant strains. During crawling on agar (Fig. 1B), all strains except for mab-20(ko) translocated significantly slower and the undulation frequency of all mutant strains was reduced. The largest reduction of speed and frequency was in $s m p-1(k o)$ animals. During swimming (Fig. 1B), plx-1(ko) and smp-1(ko) animals translocated slower and at higher undulation frequency than wild type; $s m p-2(k o)$ animals translocated slower at lower undulation frequency. On the other hand, $p / x-2(k o)$ animals translocated faster than wild type but with a lower undulation frequency. We did not observe significant locomotion differences in swimming of mab-20(ko) animals.

We focused further analysis on the plexins ( $p / x-1$ and $p / x-2)$, because as the only receptors, segregating membrane-bound and secreted pathways, they provide a comprehensive and specific manipulation of these pathways, as well as the identity of the cellular targets (Fujii et al., 2002).

\section{Gross neuronal morphology was unaffected by the absence of PLX-1 and PLX-2.}

We imaged intact young adult plexin-knockout animals expressing pan neuronal green fluorescent protein (GFP), with emphasis on neuron-rich areas around head, tail and vulva, and particularly at the commissures of motoneurons (Fig. 2). We did not observe 
any morphological differences between mutant and wild type animals in any of these regions.

\section{Motoneuronal expression of PLX-1 and PLX-2}

We imaged transcriptional reporters for $p / x-1$ and $p / x-2$ in order to identify their neuronal expression in the ventral nerve cord (VNC). GFP under the $p / x-1 p$ promoter (Figure $3 A B$ ) was mostly expressed in non-neuronal tissue including the body-wall muscle in the head and along the body, the lateral seam cells, and vulva cells. In the nervous system, plx-1p::GFP was expressed in a few neurons in the head and in two motoneurons at the base of the tail, namely DA8 and DA9 (Figure 3B inset; also reported in Mizumoto and Shen, 2013). GFP under the $p / x-2 p$ promoter was expressed in neurons in the head and tail (Figure 3CD), as well as in four motoneuron classes in the VNC: DA, DB, and DD classes in first larval stage (L1), and VC class that develop post-embryonically in $L 2$ and older animals. The nervous system of $L 1$ includes only the DA, DB and DD classes of motoneurons (White et al., 1976, 1986) with commissures that extend from the VNC to the dorsal nerve cord on the opposite side of the animal. These commissures were the targets for microsurgery.

\section{Neurites of plexin knockout mutants regenerate more than wild type after laser microsurgery.}

We disconnected 156 commissural neurites of motoneurons of wild type and plexin knockout mutant animals with laser microsurgery (Harreguy et al., 2020). These lateral processes extend to connect the ventral and dorsal nerve cords and when multiple processes are disconnected, locomotion is impaired (Yanik et al., 2004). When we examine the same neurite after 24 hours, some regenerated by sprouting a growth cone from the proximal segment and some of those reconnected to the distal segment or the dorsal nerve cord (Fig. 4A). In the wild type, 38 of 73 neurites regenerated $(0.52 \pm 0.11)$ and only 5 of the $38(13 \%)$ reconnected (Fig. 4B). The plexin knockout mutants exhibited significantly more regeneration $(p=0.049$, Fisher Exact on $2 x 3$ contingency table), 33 of $47(0.7 \pm 0.11)$ for $p / x-1(k o)$ and 26 of $36(0.72 \pm 0.12)$ for $p / x-2(k o)$. Reconnection happened significantly more $(p<0.0001$ Fisher Exact on 2x3 contingency table) in plx-2(ko): 20 of the 26 regenerated neurites, which are $77 \%$. In plx-1(ko), 13 of 33 regenerated neurites reconnected (39\%). Six hours after microsurgery, wild type and mutant animals moved slower than sham-treated animals of the same genotype. Subsequently, 24 hours after microsurgery, mean locomotion speed has recovered to levels comparable to sham-treated animals for all groups (Fig. 4C).

\section{Discussion}

Here we have demonstrated that the two plexins that mediate semaphorin signaling in C. elegans restrict neuronal regeneration and reconnection after injury. In their absence, injured neurons of plexin knockout mutants exhibit higher levels of regeneration (for both plexins) and reconnection (for $p / x-2$ ).

By the nature of their ligands, the two plexins mediate different spatial signals.

Paracrine interaction, such as those mediated by PLX-1 typically act at short-ranged by cell to cell interactions and conform subcellular resolution spatial information (Dalpé et 
al., 2004, 2005; Gurrapu and Tamagnone, 2016). Because both ligand and receptor are transmembrane proteins, the flow of information could be bidirectional, such as in the case of reverse-signaling through semaphorins, in which plexins function as ligands (Yu et al., 2010; Battistini and Tamagnone, 2016). On the other hand, juxtacrine interactions, such as those mediated by PLX-2 are spatially more disperse over tissue level where the ligand typically diffuses to set meaningful concentration gradients (Chen et al., 2007).

We demonstrated that neither the plexins nor the three semaphorins are necessary for gross neuromorphogenesis. However, at low penetrance their omission causes defasciculating and axon misguidance (Roy et al., 2000). In the nervous system, PLX-1 is only expressed in two motoneurons, namely DA8 and DA9, where it is involved in synaptic tiling during development by restricting the synaptic regions (Mizumoto and Shen, 2013). Because, to the most part, PLX-1 is expressed in muscle, seam cells, and other non-neuronal tissue (Fujii et al., 2002), we hypothesize that its restrictive effect on regeneration is achieved by interaction with the semaphorin SMP-1 presented by the motoneurons (Liu et al., 2005). The neurons could respond indirectly to the surrounding tissue via another signaling pathway, or SMP-1 could mediate a direct cellular response via reverse-signaling from plexins to semaphorins (Yu et al., 2010; Battistini and Tamagnone, 2016). The other membrane-bound semaphorin, SMP-2, might not be involved in motoneuronal regeneration because it is not expressed in VNC motoneurons, but in body wall muscle and some sensory neurons in the head (Ginzburg et al., 2002). PLX-2 is expressed in four classes of motoneurons and the most parsimonious hypothesis is that MAB-20 secretion from the laterally positioned seam cells generate a gradient that suppresses overgrowth of neurites in health and injury. A similar system was described for regenerating axons of murine spinal cord and brain, where SEMA3A and Plexin expression increases after injury and SEMA3A secreted by cells adjacent to the injury site create an exclusion zone which regenerating axons do not penetrate (Pasterkamp and Verhaagen, 2001; Pasterkamp et al., 2001; de Winter et al., 2002).

The phenotypes we describe for uninjured plexin and semaphorin knockout mutant animals are changes in speed and frequency of locomotion on agar surface and in liquid. To the most part, these effects are small in magnitude and include both increases and decreases compared to wild type animals. The largest effects were on the translocation speed of smp-1(ko) during swimming and even worse during crawling. Because the semaphorin signaling pathways are involved in several aspects of embryonic development and its components are expressed in neuronal and nonneuronal tissue in the embryo, the phenotypes are likely the product of an accumulation of effects on structure and function of different tissue, such as muscle, cuticle, or the nervous system. To remove the effect of these locomotion phenotypes from our study, we compare animals after laser microsurgery to sham-operated animals of the same genotype. Moreover, the laser microsurgery experiments included only plexin knockout mutants and smp-1(ko) animals were not included in that comparison.

Locomotion behavior was impaired 6 hours post-injury and recovered back to pre-injury parameters 24 hours post-injury in wild type animals and both plexin knockout mutant animals. Because less than half of the neurites in the wild type animals regenerated and 
only $7 \%$ reconnected, we hypothesize that the recovery is due to reorganization of the locomotion circuit to produce a meaningful motor pattern that is indistinguishable from that of an uninjured animal (Haspel et al., 2021). Similarly, the recovery of plexin knockout mutants that exhibit much higher levels of regeneration (for both) and reconnection (for plx-2) can be due to reorganization. Full recovery of locomotion with only partial recovery of neurites and synapses has been described in other systems (Oliphint et al., 2010), but the underlying circuit mechanism is unknown.

The conserved but concise semaphorin-plexin system and readily available genetic and transgenic tools in $C$. elegans, together with accurate injury and quick neuroregeneration and recovery of behavior provide an attractive experimental model. The secreted and membrane-bound semaphorin signaling pathways both restrict regeneration but in distinct processes that likely include spatial specificity and recurrent signals. Further studies, including of the localization of each of the semaphorins, before and right after injury, as well as the spatiotemporal dynamics of related secondary messengers such as calcium and cGMP, will answer fundamental questions about the involvement of semaphorin signaling in neural recovery from injury.

\section{Acknowledgements}

We thank Dr Joseph Culotti (University of Toronto, Mt Sinai Hospital) and Dr Ricard Ikegami (UC Berkeley) for sharing the $p / x-2$ and $p / x-1$ reporter strains. We also thank Dr Kang Shen (Stanford University) and Dr Kota Mizumoto (University of British Columbia) for the plx-1 reporter constructs. We thank Dr Monica Driscoll (Rutgers University) for her help in generating transgenic lines and the Hobert Lab (Columbia University) for the NeuroPal strains. Finally, we thank the State of New Jersey Commission on Spinal Cord Research for partially funding this study by grant CSCR14ERG002 to G.H. and CSCR16IRG013 to T.S.T., and the NJIT Undergraduate Research Initiative (URI) for their funding. Some strains were provided by the CGC, which is funded by NIH Office of Research Infrastructure Programs (P40 OD010440). 


\section{References}

Alto, L. T., and Terman, J. R. (2017). Semaphorins and their signaling mechanisms. Methods in Molecular Biology 1493, 1-25. doi:10.1007/978-1-4939-6448-2_1.

Assous, M., Martinez, E., Eisenberg, C., Shah, F., Kosc, A., Varghese, K., et al. (2019). Neuropilin 2 Signaling Mediates Corticostriatal Transmission, Spine Maintenance, and Goal-Directed Learning in Mice. Journal of Neuroscience 39, 8845-8859. doi:10.1523/JNEUROSCI.1006-19.2019.

Bagri, A., Cheng, H.-J., Yaron, A., Pleasure, S. J., and Tessier-Lavigne, M. (2003). Stereotyped Pruning of Long Hippocampal Axon Branches Triggered by Retraction Inducers of the Semaphorin Family. Cell 113, 285-299. doi:10.1016/S00928674(03)00267-8.

Battistini, C., and Tamagnone, L. (2016). Transmembrane semaphorins, forward and reverse signaling: have a look both ways. Cellular and molecular life sciences: CMLS 73, 1609-1622. doi:10.1007/S00018-016-2137-X.

Chen, G., Sima, J., Jin, M., Wang, K., Xue, X., Zheng, W., et al. (2007). Semaphorin-3A guides radial migration of cortical neurons during development. Nature Neuroscience 2008 11:1 11, 36-44. doi:10.1038/nn2018.

Chen, L., Wang, Z., Ghosh-Roy, A., Hubert, T., Yan, D., O'Rourke, S., et al. (2011). Axon regeneration pathways identified by systematic genetic screening in $\mathrm{C}$. elegans. Neuron 71, 1043-1057. doi:10.1016/j.neuron.2011.07.009.

Chisholm, A. D., Hutter, H., Jin, Y., and Wadsworth, W. G. (2016). The Genetics of Axon Guidance and Axon Regeneration in Caenorhabditis elegans. Genetics 204, 849 LP - 882. doi:10.1534/genetics.115.186262.

Dalpé, G., Brown, L., and Culotti, J. G. (2005). Vulva morphogenesis involves attraction of plexin 1-expressing primordial vulva cells to semaphorin 1a sequentially expressed at the vulva midline. Development 132, 1387-1400. doi:10.1242/DEV.01694.

Dalpé, G., Zhang, L., Zheng, H., and Culotti, J. (2004). Conversion of cell movement responses to Semaphorin-1 and Plexin-1 from attraction to repulsion by lowered levels of specific RAC GTPases in C. elegans. Development (Cambridge, England) 131, 2073-2088. doi:10.1242/DEV.01063.

Dalpe, G., Zheng, H., Brown, L., and Culotti, J. (2012). Semaphorin-1 and netrin signal in parallel and permissively to position the male ray 1 sensillum in Caenorhabditis elegans. Genetics 192, 959-971. doi:10.1534/genetics.112.144253.

Danelon, V., Goldner, R., Martinez, E., Gokhman, I., Wang, K., Yaron, A., et al. (2020). Modular and Distinct Plexin-A4/FARP2/Rac1 Signaling Controls Dendrite Morphogenesis. Journal of Neuroscience 40, 5413-5430. doi:10.1523/JNEUROSCI.2730-19.2020.

de Winter, F., Oudega, M., Lankhorst, A. J., Hamers, F. P., Blits, B., Ruitenberg, M. J., et al. (2002). Injury-induced class 3 semaphorin expression in the rat spinal cord. Experimental neurology 175, 61-75. doi:10.1006/exnr.2002.7884. 
Eisenberg, C., Subramanian, D., Afrasiabi, M., Ziobro, P., Delucia, J., Hirschberg, P. R., et al. (2021). Reduced hippocampal inhibition and enhanced autism-epilepsy comorbidity in mice lacking neuropilin 2. doi:10.1038/s41398-021-01655-6.

Fard, D., and Tamagnone, L. (2021). Semaphorins in health and disease. Cytokine \& growth factor reviews 57, 55-63. doi:10.1016/J.CYTOGFR.2020.05.006.

Fujii, T., Nakao, F., Shibata, Y., Shioi, G., Kodama, E., Fujisawa, H., et al. (2002). Caenorhabditis elegans PlexinA, PLX-1, interacts with transmembrane semaphorins and regulates epidermal morphogenesis. Development 129, 2053 LP - 2063. Available at: http://dev.biologists.org/content/129/9/2053.abstract.

Limoni, G. (2021). Modelling and Refining Neuronal Circuits with Guidance Cues: Involvement of Semaphorins. International journal of molecular sciences 22. doi:10.3390/IJMS22116111.

Gherardi, E., Love, C., Esnouf, R., and Jones, E. Y. (2004). The sema domain. Current Opinions in Structural Biology 14, 669-678. doi:10.1016/j.sbi.2004.10.010.

Ghosh-Roy, A., and Chisholm, A. D. (2010). Caenorhabditis elegans: a new model organism for studies of axon regeneration. Developmental dynamics : an official publication of the American Association of Anatomists 239, 1460-1464. doi:10.1002/dvdy.22253.

Ginzburg, V. E., Roy, P. J., and Culotti, J. G. (2002). Semaphorin 1a and semaphorin $1 \mathrm{~b}$ are required for correct epidermal cell positioning and adhesion during morphogenesis in C. elegans. Development 129, 2065 LP - 2078. Available at: http://dev.biologists.org/content/129/9/2065.abstract.

Gurrapu, S., and Tamagnone, L. (2016). Transmembrane semaphorins: Multimodal signaling cues in development and cancer. Cell Adhesion \& Migration 10, 675. doi:10.1080/19336918.2016.1197479.

Harreguy, M. B., Marfil, V., Grooms, N. W. F., Gabel, C. v., Chung, S. H., and Haspel, G. (2020). Ytterbium-doped fibre femtosecond laser offers robust operation with deep and precise microsurgery of C. elegans neurons. Scientific Reports 10, 1-7. doi:10.1038/s41598-020-61479-0.

Harreguy, M. B., Tran, T. S., and Haspel, G. (2021). "Neuronal microsurgery with an Ybdoped fiber femtosecond laser," in C. elegans Methods and Applications. (New york: Springer Nature). doi:10.1007/978-1-0716-2180-6_17.

Hashimoto, M., Ino, H., Koda, M., Murakami, M., Yoshinaga, K., Yamazaki, M., et al. (2004). Regulation of semaphorin 3A expression in neurons of the rat spinal cord and cerebral cortex after transection injury. Acta neuropathologica 107, 250-256. doi:10.1007/s00401-003-0805-z.

Haspel, G., Severi, K. E., Fauci, L. J., Cohen, N., Tytell, E. D., and Morgan, J. R. (2021). Resilience of neural networks for locomotion. The Journal of Physiology 599, 3825-3840. doi:10.1113/JP279214. 
Ho, J., Tumkaya, T., Aryal, S., Choi, H., and Claridge-Chang, A. (2019). Moving beyond $P$ values: data analysis with estimation graphics. Nature Methods. doi:10.1038/s41592-019-0470-3.

Ikegami, R., Simokat, K., Zheng, H., Brown, L., Garriga, G., Hardin, J., et al. (2012). Semaphorin and Eph receptor signaling guide a series of cell movements for ventral enclosure in C. elegans. Current biology : CB 22, 1-11. doi:10.1016/j.cub.2011.12.009.

Javer, A., Ripoll-Sánchez, L., and Brown, A. E. X. (2018). Powerful and interpretable behavioural features for quantitative phenotyping of Caenorhabditis elegans. Philosophical transactions of the Royal Society of London. Series B, Biological sciences 373, 20170375. doi:10.1098/rstb.2017.0375.

Junqueira Alves, C., Yotoko, K., Zou, H., and Friedel, R. H. (2019). Origin and evolution of plexins, semaphorins, and Met receptor tyrosine kinases. Scientific Reports 2019 9:1 9, 1-14. doi:10.1038/s41598-019-38512-y.

Kaneko, S., Iwanami, A., Nakamura, M., Kishino, A., Kikuchi, K., Shibata, S., et al. (2006). A selective Sema3A inhibitor enhances regenerative responses and functional recovery of the injured spinal cord. Nature Medicine 2006 12:12 12, 1380-1389. doi:10.1038/nm1505.

Kolodkin, A., and Tessier-Lavigne, M. (2011). Mechanisms and molecules of neuronal wiring: a primer. Cold Spring Harbor perspectives in biology 3, 1-14. doi:10.1101/CSHPERSPECT.A001727.

Koropouli, E., and Kolodkin, A. L. (2014). Semaphorins and the dynamic regulation of synapse assembly, refinement, and function. Current Opinion in Neurobiology 27, 1-7. doi:10.1016/J.CONB.2014.02.005.

Liu, Z., Fujii, T., Nukazuka, A., Kurokawa, R., Suzuki, M., Fujisawa, H., et al. (2005). C. elegans PlexinA PLX-1 mediates a cell contact-dependent stop signal in vulval precursor cells. Developmental biology 282, 138-151.

doi:10.1016/j.ydbio.2005.03.002.

Lyons, L. C., and Hecht, R. M. (1997). Acute ethanol exposure induces nondisjunction of the X chromosome during spermatogenesis. Worm Breeders Gazette 14, 52.

Melentijevic, I., Toth, M. L., Arnold, M. L., Guasp, R. J., Harinath, G., Nguyen, K. C., et al. (2017). C. elegans neurons jettison protein aggregates and mitochondria under neurotoxic stress. Nature 542, 367-371. doi:10.1038/nature21362.

Mizumoto, K., and Shen, K. (2013). Interaxonal interaction defines tiled presynaptic innervation in C. elegans. Neuron 77, 655-666. doi:10.1016/j.neuron.2012.12.031.

Mlechkovich, G., Peng, S.-S., Shacham, V., Martinez, E., Gokhman, I., Minis, A., et al. (2014). Distinct cytoplasmic domains in Plexin-A4 mediate diverse responses to semaphorin $3 \mathrm{~A}$ in developing mammalian neurons. Science signaling 7 , ra24. doi:10.1126/scisignal.2004734. 
Nakao, F., Hudson, M. L., Suzuki, M., Peckler, Z., Kurokawa, R., Liu, Z., et al. (2007). The PLEXIN PLX-2 and the ephrin EFN-4 have distinct roles in MAB20/Semaphorin 2A signaling in Caenorhabditis elegans morphogenesis. Genetics 176, 1591-1607. doi:10.1534/genetics.106.067116.

Negishi, M., Oinuma, I., and Katoh, H. (2005). Review Plexins: axon guidance and signal transduction. CMLS, Cell. Mol. Life Sci 62, 1363-1371. doi:10.1007/s00018005-5018-2.

Neumann, B., Nguyen, K. C. Q., Hall, D. H., Ben-Yakar, A., and Hilliard, M. A. (2011). Axonal regeneration proceeds through specific axonal fusion in transected $\mathrm{C}$. elegans neurons. Developmental Dynamics 240, 1365-1372. doi:10.1002/dvdy.22606.

Oliphint, P. A., Alieva, N., Foldes, A. E., Tytell, E. D., Lau, B. Y.-B., Pariseau, J. S., et al. (2010). Regenerated Synapses in Lamprey Spinal Cord Are Sparse and Small Even After Functional Recovery From Injury. The Journal of comparative neurology 518, 2854. doi:10.1002/CNE.22368.

Pascoe, H. G., Wang, Y., and Zhang, X. (2015). Structural mechanisms of plexin signaling. Progress in Biophysics and Molecular Biology 118. doi:10.1016/j.pbiomolbio.2015.03.006.

Pasterkamp, R., Anderson, P., and Verhaagen, J. (2001). Peripheral nerve injury fails to induce growth of lesioned ascending dorsal column axons into spinal cord scar tissue expressing the axon repellent Semaphorin3A. The European journal of neuroscience 13, 457-471. doi:10.1046/J.0953-816X.2000.01398.X.

Pasterkamp, R. J., and Verhaagen, J. (2001). Emerging roles for semaphorins in neural regeneration. Brain research. Brain research reviews 35, 36-54.

Riccomagno, M., and Kolodkin, A. (2015). Sculpting neural circuits by axon and dendrite pruning. Annual review of cell and developmental biology 31, 779-805. doi:10.1146/ANNUREV-CELLBIO-100913-013038.

Riccomagno, M. M., Hurtado, A., Wang, H., MacOpson, J. G. J., Griner, E. M., Betz, A., et al. (2012). The RacGAP $\beta 2-C h i m a e r i n$ Selectively Mediates Axonal Pruning in the Hippocampus. Cell 149, 1594-1606. doi:10.1016/J.CELL.2012.05.018.

Roy, P. J., Zheng, H., Warren, C. E., and Culotti, J. G. (2000). mab-20 encodes Semaphorin-2a and is required to prevent ectopic cell contacts during epidermal morphogenesis in Caenorhabditis elegans. Development 127, 755-767. doi:10.1242/DEV.127.4.755.

Shen, K., and Cowan, C. (2010). Guidance molecules in synapse formation and plasticity. Cold Spring Harbor perspectives in biology 2. doi:10.1101/CSHPERSPECT.A001842.

Spinelli, E. D., McPhail, L. T., Oschipok, L. W., Teh, J., and Tetzlaff, W. (2007). Class A plexin expression in axotomized rubrospinal and facial motoneurons. Neuroscience 144, 1266-1277. doi:10.1016/J.NEUROSCIENCE.2006.10.057. 
Stiernagle, T. (2006). Maintenance of C. elegans. WormBook: the online review of C. elegans biology, 1-11. doi:10.1895/wormbook.1.101.1.

Tamagnone, L., Artigiani, S., Chen, H., He, Z., Ming, G. L., Song, H. J., et al. (1999). Plexins Are a Large Family of Receptors for Transmembrane, Secreted, and GPIAnchored Semaphorins in Vertebrates. Cell 99, 71-80. doi:10.1016/S00928674(00)80063-X.

Tran, T. S., Rubio, M. E., Clem, R. L., Johnson, D., Case, L., Tessier-Lavigne, M., et al. (2009). Secreted semaphorins control spine distribution and morphogenesis in the postnatal CNS. Nature 462, 1065-1069. doi:10.1038/nature08628.

White, J. G., Southgate, E., Thomson, J. N., and Brenner, S. (1976). The structure of the ventral nerve cord of Caenorhabditis elegans. Philosophical Transactions of the Royal Society of London. B, Biological Sciences 275, 327-348.

doi:10.1098/RSTB.1976.0086.

White, J. G., Southgate, E., Thomson, J. N., and Brenner, S. (1986). The structure of the nervous system of the nematode Caenorhabditis elegans. Philosophical transactions of the Royal Society of London. Series B, Biological sciences 314, 1340. doi:10.1098/rstb.1986.0056.

Yanik, M. F., Cinar, H. N. H., Cinar, H. N. H., Chisholm, A. D., Jin, Y., and Ben-Yakar, A. (2004). Functional regeneration after laser axotomy. Nature 432, 822. doi:10.1038/432822a https://www.nature.com/articles/432822a\#supplementaryinformation.

Yaron, A., Huang, P., Cheng, H., and Tessier-Lavigne, M. (2005). Differential requirement for Plexin-A3 and $-\mathrm{A} 4$ in mediating responses of sensory and sympathetic neurons to distinct class 3 Semaphorins. Neuron 45, 513-523. doi:10.1016/J.NEURON.2005.01.013.

Yoshida, Y. (2012). Semaphorin signaling in vertebrate neural circuit assembly. Frontiers in molecular neuroscience 5, 71. doi:10.3389/fnmol.2012.00071.

Yu, L., Zhou, Y., Cheng, S., and Rao, Y. (2010). Plexin A-Semaphorin-1a Reverse Signaling Regulates Photoreceptor Axon Guidance in Drosophila. The Journal of Neuroscience 30, 12151. doi:10.1523/JNEUROSCI.1494-10.2010.

Zhou, X., Wahane, S., Friedl, M.-S., Kluge, M., Friedel, C. C., Avrampou, K., et al. (2020). Microglia and macrophages promote corralling, wound compaction and recovery after spinal cord injury via Plexin-B2. Nature Neuroscience 2020 23:3 23, 337-350. doi:10.1038/s41593-020-0597-7. 


\section{Figures}
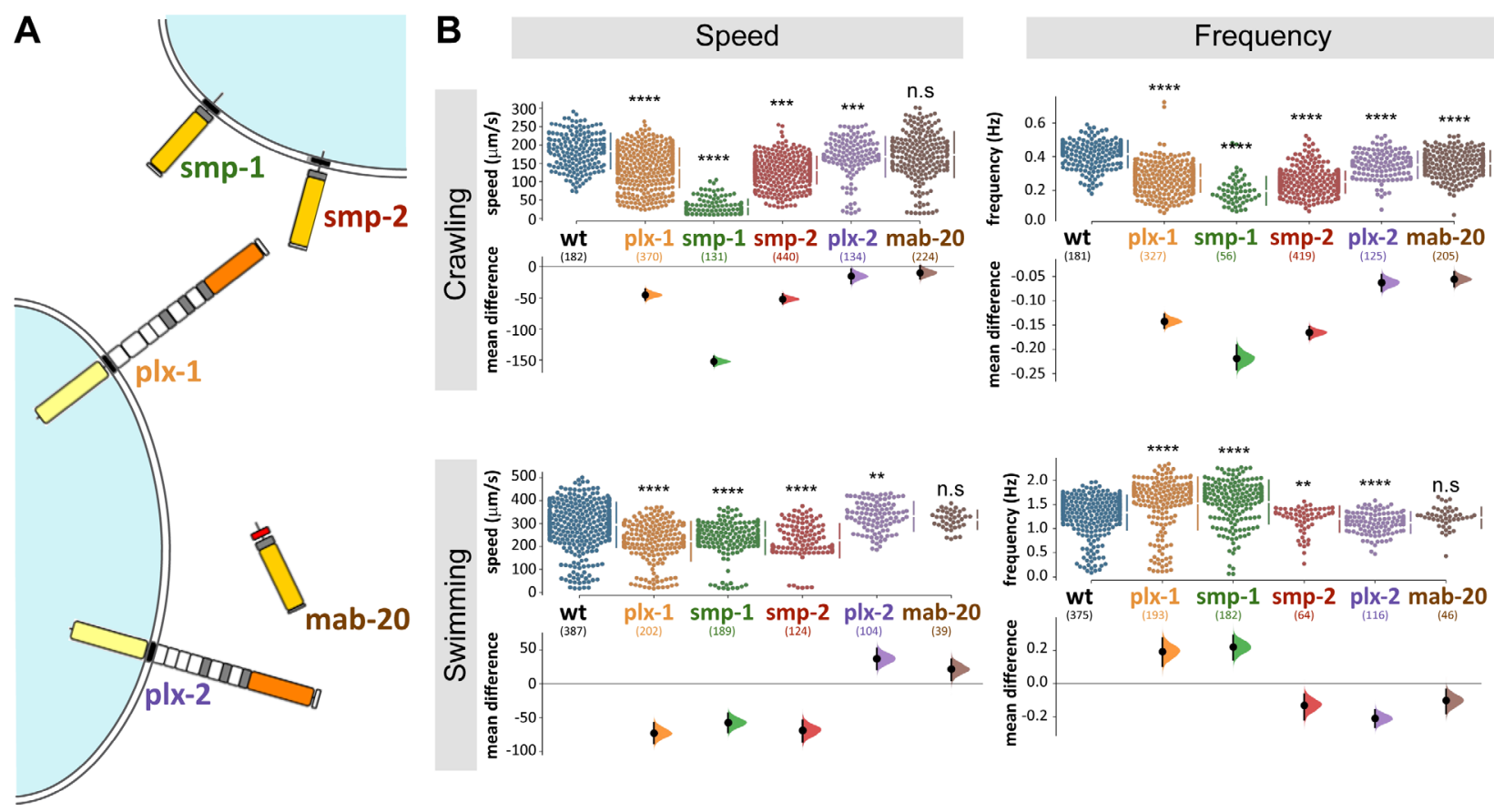

Figure 1. C. elegans semaphorin system comprises only three ligands and two receptors and omitting any one component affects locomotion. A) Semaphorin signaling system of $C$. elegans. The membrane bound semaphorins $s m p-1$ and $s m p-2$ signal through $p / x-1$, while the secreted mab-20 signals through plx-2 (molecular diagrams adapted from Junqueira Alves et al., 2019). B) Mutant strains with knocked out semaphorins or plexins are significantly different from wild type when crawling (locomoting on agar) or swimming (locomoting in liquid media). The largest difference was in $s m p-1$ (ko) animals while mab-2O(ko) animals are only different for lower undulatory frequency during crawling. Data points are mean absolute translocation speed or frequency to both direction of locomotion of analyzed trajectories; n.s. $p>0.05,{ }^{* *} p<0.01,{ }^{* * *} p<0.001,{ }^{* * * *} p<0.0001$; two-sided permutation t-test; in parentheses are the number of analyzed trajectories from 20 25 animals. 


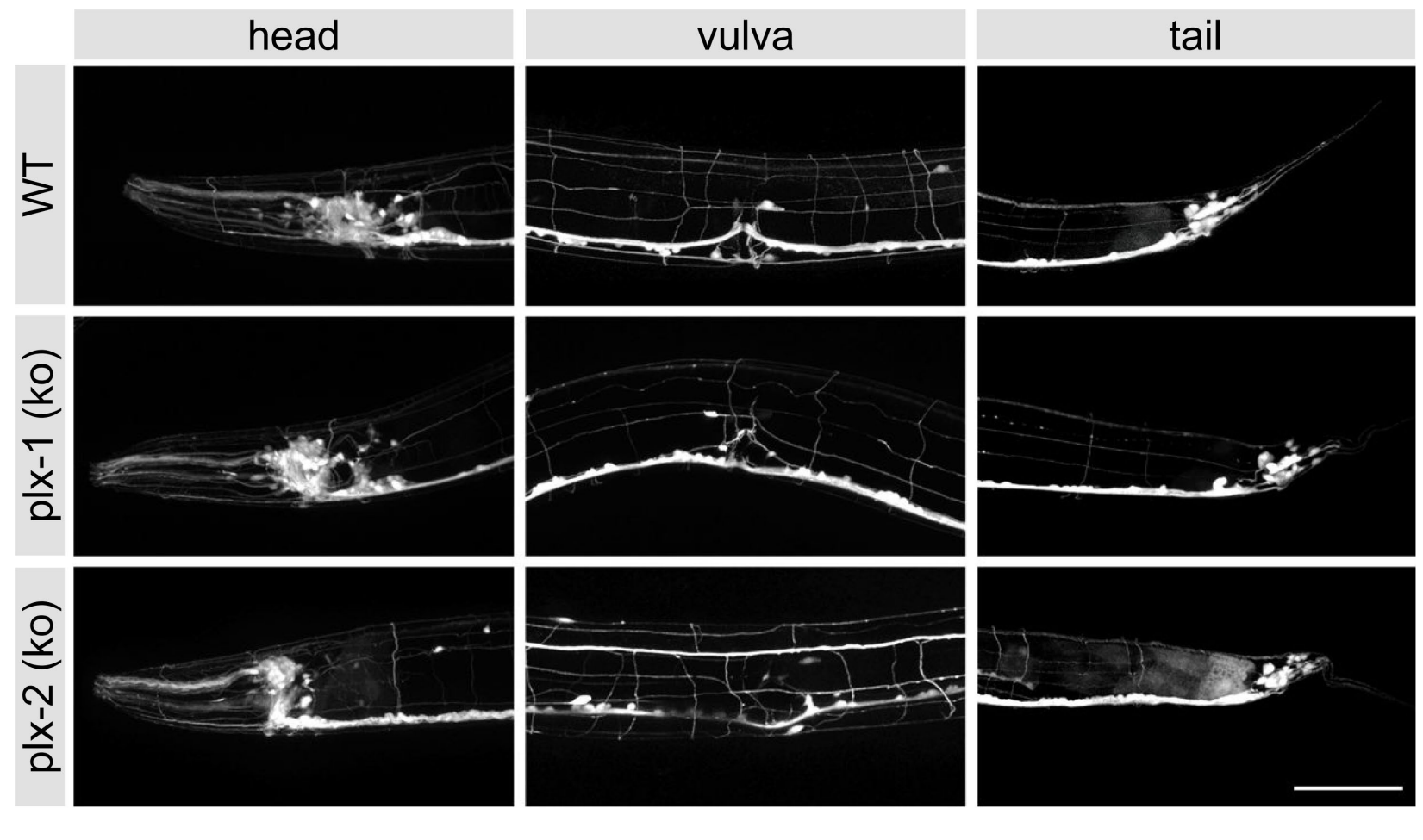

Figure 2. Neuronal morphology of plexin knockout strains is comparable to wild type. We imaged pan-neuronal GFP in selected neuron-rich areas (vulva, nose, and tail) of wild type (WT) and knockout mutant animals ( $p / x-1$ (ko) and $p / x-$ $2(\mathrm{ko})$ ), as well as the entire animals (not shown), to look for gross neuromorphological differences. We did not observe differences between wild type and mutant strains. $N=5$ animals for each strain. Scale bar $=50 \mu \mathrm{m}$. 

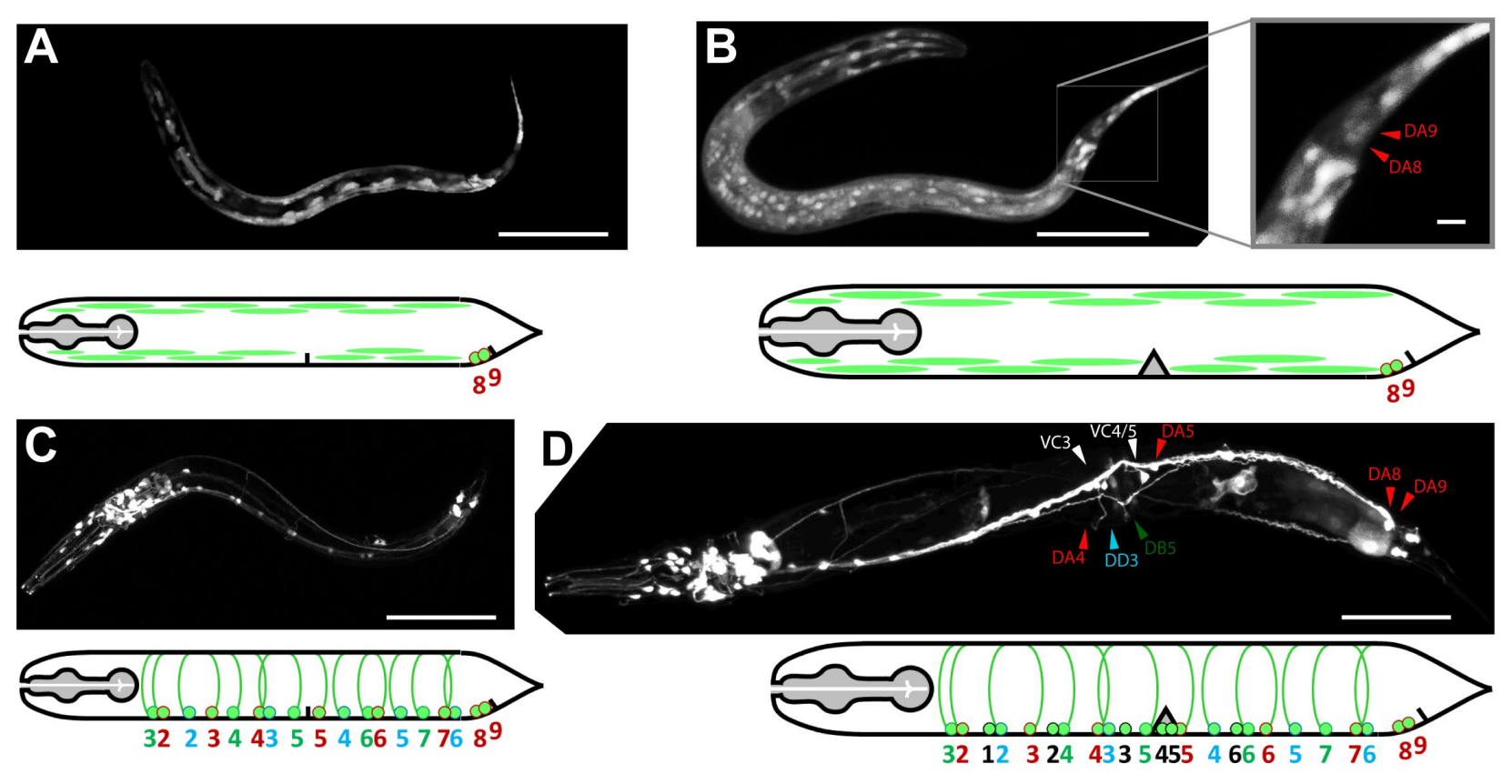

Figure 3. PLX-1 is expressed in DA8, DA9, and in non-neuronal tissue, while PLX-2 is expressed in dorsal excitatory and inhibitory motoneurons. $A B$ ) Green fluorescent protein (GFP) driven by $p / x-1 p$ promoter expressed in nonneuronal tissue such as body-wall muscle, seam cells and in two motoneurons close to the tail: DA8 and DA9 (inset, red arrowhead). C) GFP driven by $p / x-1 p$ promoter expressed in all three motoneuron classes in the L1 larvae: DA, DB, and DD (two excitatory and one inhibitory dorsal motoneurons). D) In older animals, GFP was also expressed in VC neurons. Colored numbers in diagrams specify names of motoneurons at that location: DA (red), DB (green), DD (blue), and VC (black). All panel scale bars are $50 \mu \mathrm{m}$; inset scale bar $5 \mu \mathrm{m}$. 

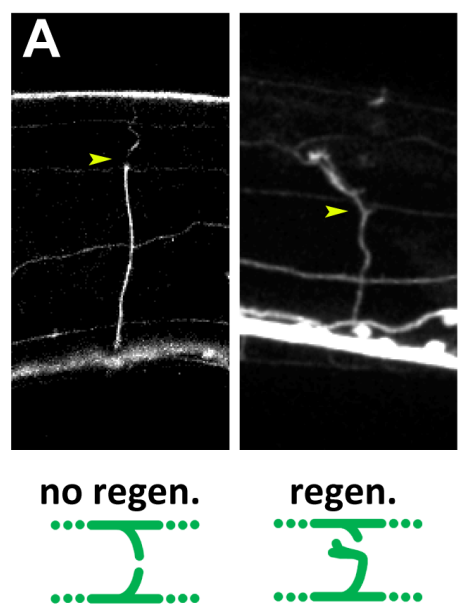

regen.
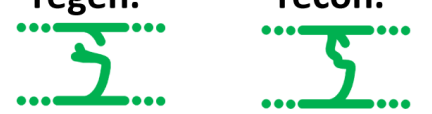

$B$

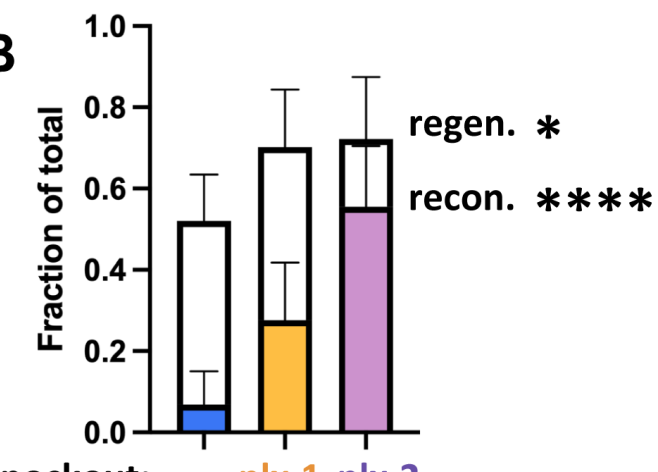

knockout: - plx-1 plx-2

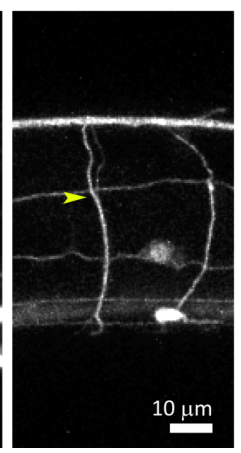

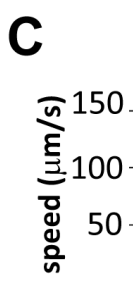

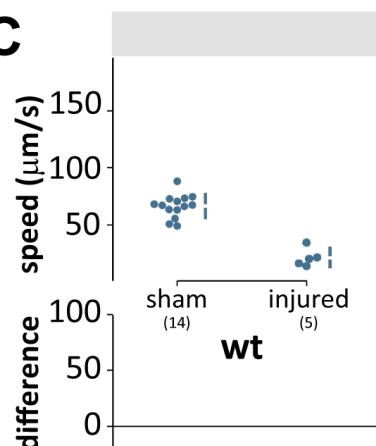

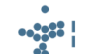
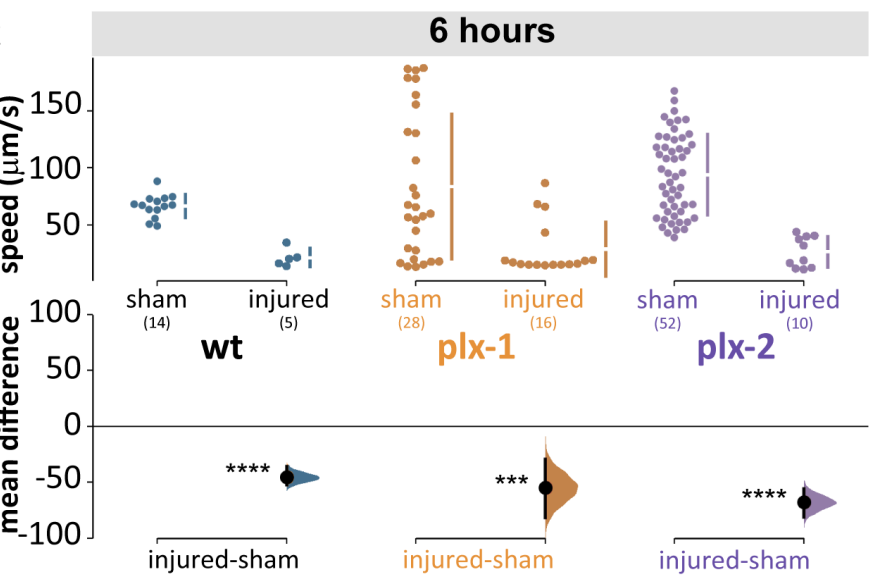

24 hours

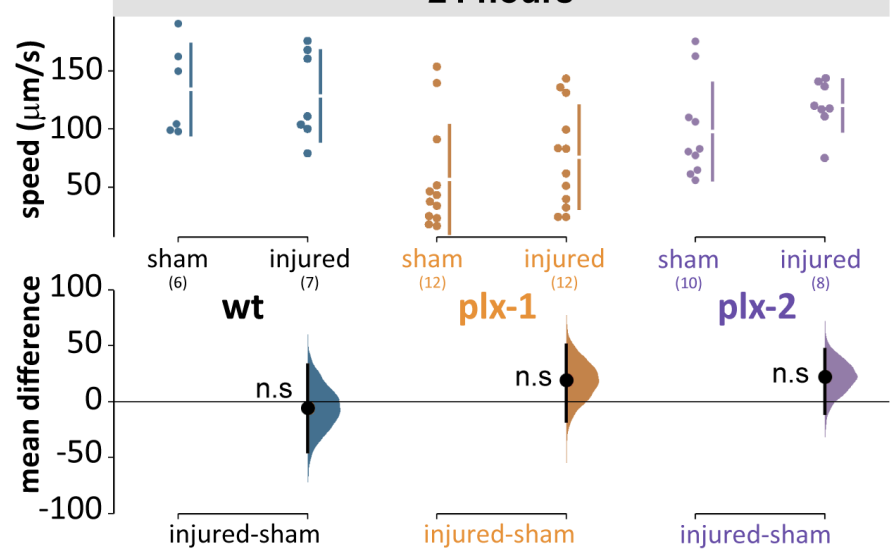

Figure 4. Neuronal regeneration and reconnection increased in the absence of plexins $\mathbf{2 4}$ hours after laser microsurgery, while locomotion speed fully recovers in all genotypes. A) We scored all commissural neurites 24 hours after microsurgery (yellow arrowhead) and scored them as exhibiting no-regeneration, regeneration, or reconnection (green diagrams, see methods). B) About half of wild type neurites regenerated $24 \mathrm{~h}$ post-injury and only $7 \%$ reconnected. Both plexin knockout mutant strains exhibited more regeneration (sum of colorful and empty bars) and $p / x-2$ exhibited more reconnection (colorful bars). C) Animals of all groups moved significantly slower 6 $\mathrm{h}$ post-injury and all recovered to levels equal to sham operated after $24 \mathrm{~h}$. Data points are mean absolute translocation speed to both direction of locomotion; n.s. $p>0.05$, ${ }^{*} p<0.05{ }^{* * *} p<0.001,{ }^{* * *} p<0.0001$; two-sided permutation t-test; in parentheses are the number of analyzed trajectories from 20-25 animals. 